J. Lake Sci. (湖泊科学) , 2016, 28(5): 1018-1022

DOI 10. 18307/2016. 0511

(C) 2016 by Journal of Lake Sciences

\title{
离子交换法处理含砷废水的小试/中试试验”
}

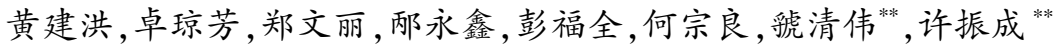

(环境保护部华南环境科学研究所,广州 510655)

\begin{abstract}
摘 要: 利用选择性复合树脂处理含砷废水, 使出水水质达到国家《地表水环境质量标准》(GB 3838-2002), 同时从选 择性复合树脂和阳离子柱上交换下来的离子可以生成 $\mathrm{H}_{2} \mathrm{O}$ 和中性盐类, 无二次污染. 研究采用 $201 \times 7$ 苯乙烯系强碱凝胶 型树脂 (I) 和 D301 大孔妈碱阴离子交换树脂( II) 作为比选树脂,通过小试试验选择具有较高交换容量的 $201 \times 7$ 苯乙 烯系强碱凝胶型树脂用于放大规模的现场中试. 实验所用选择性复合树脂制作成本及整个中试系统价格低廉, 适合大规 模工厂应用.
\end{abstract}

关键词: 砷;离子交换; 中试; 泉涌水

\section{Treatment of arsenic wastewater by ion-exchange resin}

HUANG Jianhong, ZHUO Qiongfang, ZHENG Wenli, BING Yongxin, PENG Fuquan, HE Zongliang, GUO Qingwei $^{* *} \&$ XU Zhencheng ${ }^{* *}$

(South China Institute of Environmental Science, Ministry of Environmental Protection, Guangzhou 510655, P.R.China)

\begin{abstract}
The selective composite resins were used to treat spring water containing arsenic, and the arsenic concentration in the effluent reached the Environmental Quality Standards for Surface Water (GB 3838 - 2002). Ions exchanged from the selective composite resin and cation exchange column have yielded $\mathrm{H}_{2} \mathrm{O}$ and neutral salt, and this method has no secondary pollution. $201 \times 7$ gel type styrene of strong akali resin ( I ) and D301 macroporous have weakened the akali anionic exchange resin ( II ), when compared to choose the suitable resin for arsenic removal in this study. The $201 \times 7$ resins with higher exchange capability and lower cost were applied to the pilot scale experiments on site and they were suitable to large-scale applications.
\end{abstract}

Keywords: Arsenic; ion exchange; pilot plant experiment; spring water

砷 (As) 及其化合物广泛存在于钢铁、有色冶炼、硫酸、农药和木料防腐剂等工业生产废水中, 在砷矿丰 富且又是水稻主产区的省份, 用含砷污水灌溉会导致水稻的砷污染 ${ }^{[1]}$. 在自然界有三价无机态 $\operatorname{As}($ III $)$ 、五 价无机态 $\operatorname{As}(\mathrm{V})$ 以及有机砷 MMA (甲基胂酸)、DMA (二甲基胂酸)、TMA(三甲基砷酸) 等,具有生殖毒性、 心血管毒性、肝脏毒性危害. 近年来,由于经济利益驱使, 个别单位和个人违法作业,造成各类环境砷污染事 故不断发生, 引发严重的环境危害和健康危害 ${ }^{[2]}$. 砷在水体中主要以 +3 价和 +5 价的无机酸形式存在 ${ }^{[3]}$, 砷 污染的常规处理方法分为 3 类, 即物理法、化学法和生化法 ${ }^{[4.5]}$. 由于离子交换法产生的污泥量仅为化学沉 淀法产生污泥量的 $20 \%$, 污泥的处置费用大大减少. 而且离子交换法处理量大、操作简单、易再生、效果好, 能够达到严格的排放标准,故适合工业化生产 ${ }^{[6-7]}$. 据国内外的报道, 在对低含量含砷水的处理中, 较有成效 的有无机离子交换剂 (如水合二氧化钛, 即 $\mathrm{TiO}_{2} \cdot \mathrm{H}_{2} \mathrm{O}$ ) ${ }^{[8]}$ 和有机离子交换剂 (如经二价铜离子活化的阳离 子交换树脂和聚苯乙烯强碱型阴离子交换树脂 ${ }^{[9-10]}$. 其中有机离子交换剂聚苯乙烯强碱型阴离子交换树 脂对 $\operatorname{As}(V)$ 有良好的去除效果, 已有实际应用报道 ${ }^{[11]}$.

本研究采用选择性复合树脂,基于物理化学反应的离子交换和吸附过程,树脂表面可交换基团 $\mathrm{R}$ 吸附

* 国家水体污染控制与治理科技重大专项 (2010ZX07212-007,2012ZX07206-002,2012ZX07206-003) 和铟提取过程污 染控制与管理方案研究项目 (201309051) 联合资助. 2015-09-29 收稿;2015-11-09 收修改稿. 黄建洪( 1978 ), 男,博士, 副研究员;E-mail: huangjianhong@ scies.org.

** 通信作者;E-mail: guoqingwei@ scies.org,E-mail: xuzhencheng@ scies.org. 
原水中的 $\mathrm{AsO}_{4}^{3-}$ 和 $\mathrm{AsO}_{3}^{3-}$ 并发生交换反应, 出水总砷浓度可以达到国家《地表水环境质量标准》( $\mathrm{GB} 3838-$ 2002 ) 的 $0.05 \mathrm{mg} / \mathrm{L}$, 从选择性复合树脂交换下来的离子可以生成 $\mathrm{H}_{2} \mathrm{O}$ 和中性盐类, 无二次污染. 当树脂上所 有可用于交换的有效位置饱和时, 出水中砷的浓度将快速上升甚至超过进水中砷的浓度. 经过药剂反洗树 脂再生, 可以重复利用.

\section{1 试验材料与方法}

\section{1 试验水样}

含砷废水采用阳宗海砷污染事件中云南省锦业工贸有限公司取水泵站附近泉眼含砷泉水 (下称“泉涌 水”). 泉涌出水经周边湖水迅速稀释, 经提升原提升至厂区后浓度为 $3.200 \sim 4.200 \mathrm{mg} / \mathrm{L}$, 小试试验期间浓度 为 $3.226 \mathrm{mg} / \mathrm{L}$, 中试试验平均浓度为 $3.630 \mathrm{mg} / \mathrm{L}$.

\section{2 材料设备}

小试试验离子交换柱采用内径为 $1.5 \mathrm{~cm}$ 和 2.0 $\mathrm{cm}$ 玻璃滴定管, 所用离子交换树脂为 $201 \times 7$ 苯乙烯 系强碱凝胶型树脂 ( I ) 和 D301 大孔弱碱阴离子交 换树脂 (II). 两种树脂的理化指标如表 1 所示.

中试试验设备有: 提升葲 1 台、潜水排水泵 1 台、主体装置 1 套 (含阴、阳复合离子交换柱各 1 根)、砂滤桶 1 个、高位水槽 1 个、高位再生液储槽 1 个.

\section{3 方法}

1.3.1 小试试验 两种复合交换树脂I和II对砷去除的 工艺参数供中试试验设计参考. 实验装置如图 1 所 示, 称取 $10 \mathrm{~g} 201 \times 7$ 凝胶型树脂 (I), 按《离子交换树 脂预处理方法》( GB 5476-1996) 预处理后装人内径 为 $1.5 \mathrm{~cm}$ 的交换柱中, 树脂高度为 $10 \mathrm{~cm}$, 以 $0.44 \mathrm{ml} / \mathrm{s}$ 流量处理砷浓度为 $3.226 \mathrm{mg} / \mathrm{L}$ 的泉涌水; 称取 $10 \mathrm{~g}$ D301 大孔弱碱阴离子交换树脂 (II) 预处理后装人内 径为 $2 \mathrm{~cm}$ 的交换柱中, 树脂高约 $5.5 \mathrm{~cm}$, 以 $0.27 \mathrm{ml} / \mathrm{s}$ 的流量处理泉涌水,取样时间间隔为 $10 \mathrm{~min}$.
表 1 两种树脂的理化指标

Tab.1 Characteristics of two kinds of resin

\begin{tabular}{ccc}
\hline 理化指标 & I & II \\
\hline 含水量 $/ \%$ & $42 \sim 48$ & $50 \sim 55$ \\
质量全交换容量 1$) /(\mathrm{mmol} / \mathrm{g})$ & 3.6 & 4.2 \\
湿视密度 $/(\mathrm{g} / \mathrm{ml})$ & $0.66 \sim 0.75$ & $0.75 \sim 0.85$ \\
湿真密度 $/(\mathrm{g} / \mathrm{ml})$ & $1.06 \sim 1.11$ & $1.25 \sim 1.28$ \\
粒径 $/ \mathrm{mm}$ & $0.315 \sim 1.250$ & $0.315 \sim 1.250$ \\
粒度 $/ \%^{2)}(0.315 \sim 1.5)$ & 95 & 95 \\
磨后圆球率耐磨率 $/ \%{ }^{3)}$ & $\geqslant 95$ & $\geqslant 90$ \\
\hline
\end{tabular}

1) 质量全交换容量: 表示单位质量 (每克)干树脂中所能交 换的离子 (相当于一价离子) 的物质的量, 其标志离子交换 树脂交换能量的大小, 是衡量离子交换树脂性能的重要参 数; 2 ) 粒度 $(0.315 \% \sim 1.5 \%$ ): 指定粒径范围 (小于上限粒径 $1.5 \mathrm{~mm}$ 至大于和等于下限粒径 $0.315 \mathrm{~mm}$ ) 内试样颗粒的体 积占全部试样颗粒体积的体积分数; 3 ) 磨后圆球率耐磨率: 用瓷球的滚磨对树脂施加压力和摩擦力后将树脂烘干至能 自由滚动并经分离, 其中球状颗粒占式样的质量分数即为 磨后圆球率.

1.3.2 中试实验 将含砷泉涌水引人石英砂滤槽预处理去除悬浮物后泵入高位水槽, 然后引入串联的离子交 换柱 I 、II. 含砷泉涌水经两柱处理后, 出水总砷浓度 $<0.10 \mathrm{mg} / \mathrm{L}$. 当柱子饱和后, 先用清水反洗离子柱, 再 用相应的再生液 ( $2 \mathrm{~mol} / \mathrm{L}$ 的氢氧化钠) 进行再生处理, 初期再生反洗液含砷等重金属浓度高, 可用絮凝共沉 淀等方法处理转移到固相后按危险废物标准进行处理处置, 脱泥上清液回流到本处理工艺; 后期低砷再生 反洗液可配到新鲜再生液中. 经再生液再生完的离子柱最后用清水冲洗后进人下一个交换处理周期. 中试 试验工艺流程如图 2 所示. 中试试验期间每隔 30 60 min 对出水进行取样.

总砷分析方法为乙二胺基二硫代甲酸银分光光度法,标准曲线经砷质控溶液验证满足精度要求.

\section{2 结果与分析}

按照阳宗海砷污染治理招标要求, 处理后的泉涌水总砷浓度达到 $0.10 \mathrm{mg} / \mathrm{L}$, 因此本试验只做穿透曲线 的部分数据分析.

\section{1 小试实验}

采用 $201 \times 7$ 型树脂 I 处理泉涌水时, 在处理水样时间为 $160 \mathrm{~min}$ 内, 出水总砷浓度可达到《地表水环境 质量标准》(GB 3838-2002), 处理时间为 $250 \mathrm{~min}$ 前, 出水总砷浓度 $<0.10 \mathrm{mg} / \mathrm{L}$ ( 图 3a), 满足《农田灌溉水 水质标准》 ( GB 5084-1992). 


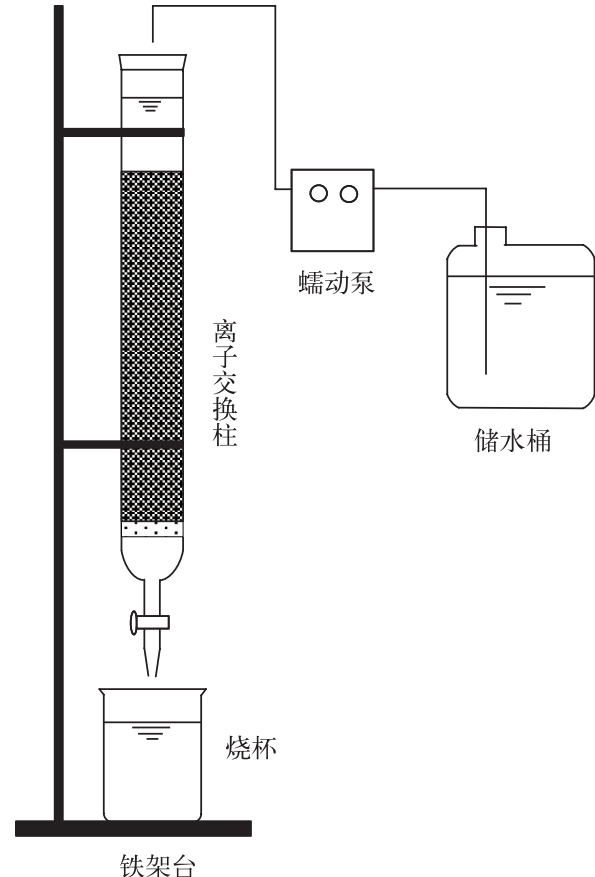

图 1 实验装置图

Fig. 1 Diagram of experiment set-up

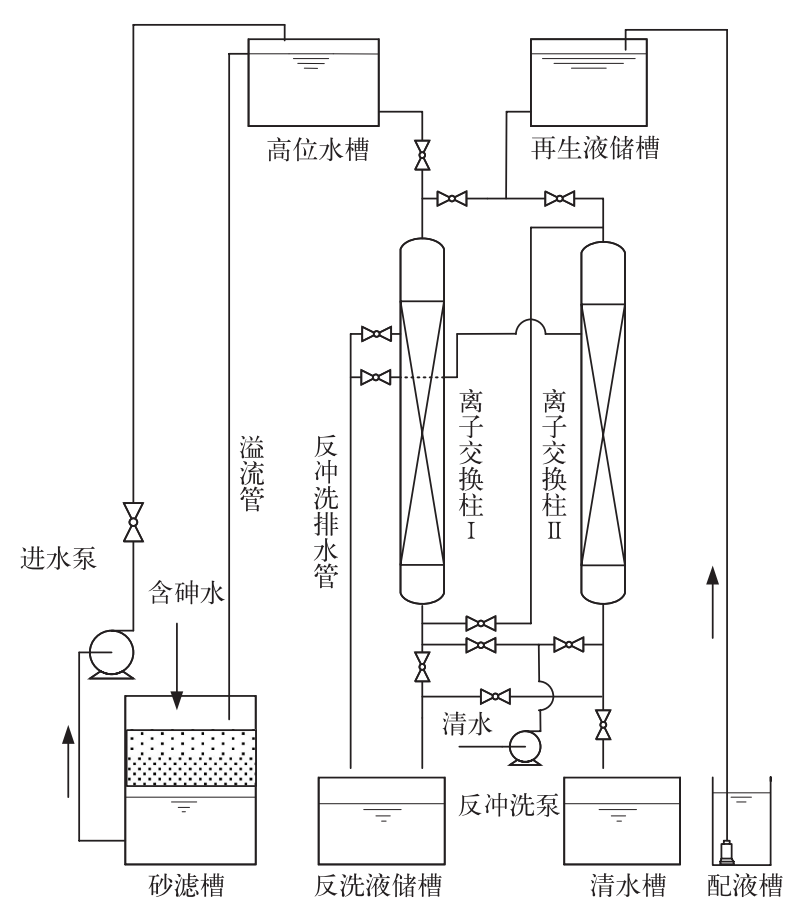

图 2 现场中试实验工艺流程

Fig.2 Process flow diagram of pilot plant experiment on site

由最小二乘法计算得出: 当去除砷 $13.52 \mathrm{mg}$ 时, 出水砷浓度将 $>0.05 \mathrm{mg} / \mathrm{L}$, 平均每克树脂去除砷 1.352 $\mathrm{mg}$; 当去除 $21.01 \mathrm{mg}$ 砷时, 出水砷浓度将 $>0.10 \mathrm{mg} / \mathrm{L}$, 平均每克树脂去除砷 $2.101 \mathrm{mg}$.

采用 D301 型阴离子交换树脂 II 处理泉涌水时, 出水水质在 $90 \mathrm{~min}$ 内可达《地表水环境质量标准》( GB 3838-2002), $190 \mathrm{~min}$ 内达《农田灌溉水水质标准》(GB 5084-1992) (图 3b).
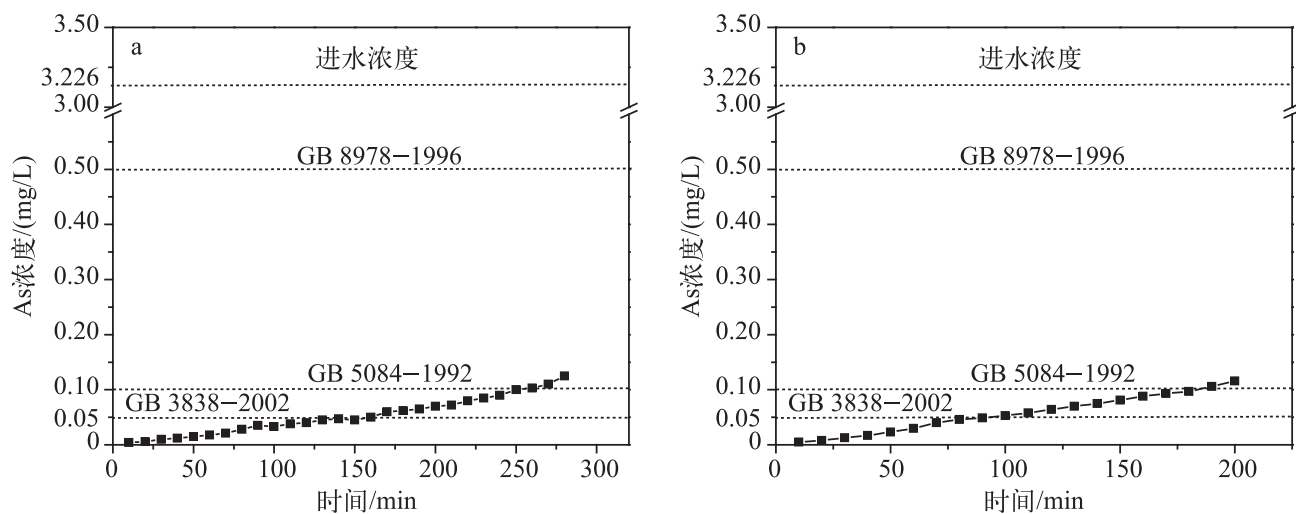

图 3 选择性复合树脂 I 和 II 的处理效果

Fig.3 Removal efficiency of selective composite resins I and II

\section{2 中试试验}

中试试验泉涌水砷浓度在 $2.99 \sim 4.47 \mathrm{mg} / \mathrm{L}$ 之间波动, 平均浓度为 $3.63 \mathrm{mg} / \mathrm{L}$. 中试试验利用树脂 $\mathrm{I}$ 处理 含砷泉水, 连续取样 $193.5 \mathrm{~h}$, 在处理时间为 $24.5 \mathrm{~h}$ 内, 出水总砷浓度 $<0.05 \mathrm{mg} / \mathrm{L}$, 达到《地表水环境质量标 
准》( GB 3838-2002); 处理时间在 $100.5 \mathrm{~h}$ 内, 出水总 砷浓度 $<0.10 \mathrm{mg} / \mathrm{L}$, 可达到《农田灌溉水水质标准》( GB 5084-1992); 当交换时间超过 $153.5 \mathrm{~h}$ 时, 出水总砷浓 度迅速增大; 当处理时间到 $168.5 \mathrm{~h}$ 时, 出水中总砷浓度 与进水中浓度相同, 表明树脂已经穿透. 之后, 出水砷 浓度超过原水浓度, 达 $4.09 \mathrm{mg} / \mathrm{L}$, 表明吸附在树脂上的 砷阴离子开始被其他离子所替代并进人水中 (图 4). 实 际应用时, 本工艺按照出水总砷浓度 $<0.10 \mathrm{mg} / \mathrm{L}$ 为界 限, 当出水砷浓度超过 $0.10 \mathrm{mg} / \mathrm{L}$ 时 (本中试工况下, 处 理时间为 $100 \mathrm{~h}$ ), 对树脂进行再生处理后重复使用. 用 $2 \mathrm{~mol} / \mathrm{L}$ 的氢氧化钠 (含 $5 \%$ 硫氢化钠) 作为洗脱液洗涤 树脂,使之恢复离子交换能力, 重新投人使用.

同样利用最小二乘法计算可得: 当去除砷 $38.479 \mathrm{~g}$ 时, 出水砷浓度将 $>0.05 \mathrm{mg} / \mathrm{L}$, 平均每克树脂去除砷

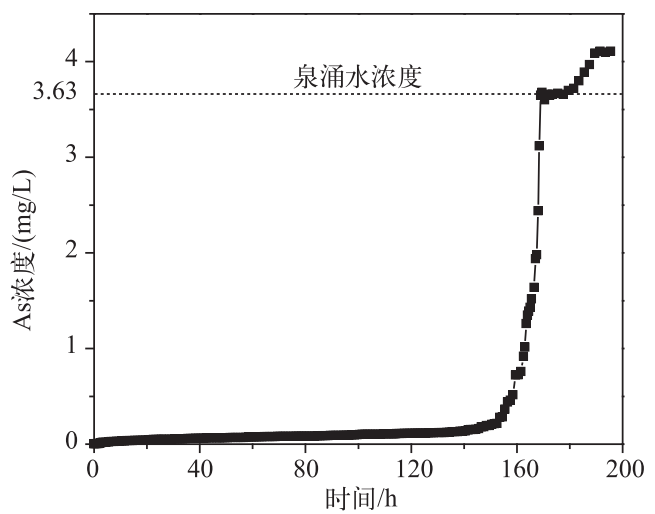

图 4 中试实验水处理效果

Fig.4 Removal efficiency of pilot plant experiment $1.283 \mathrm{mg}$; 当去除砷 $60.203 \mathrm{~g}$ 时, 出水砷浓度将>0.10 $\mathrm{mg} / \mathrm{L}$, 平均每克树脂去除砷 $2.020 \mathrm{mg}$, 表明出水砷浓度降低时, 树脂交换基团过剩; 出水砷浓度升高时, 树脂 交换基团趋于饱和. 在出水总砷浓度 $<0.10 \mathrm{mg} / \mathrm{L}$ 的时段内, 中试结果与小试结果基本吻合.

\section{3 离子交换影响因素分析}

离子交换对 $\mathrm{As}(\mathrm{V})$ 的去除能力主要取决于树脂中相邻电荷的空间距离、官能团的流动性、伸展性以及 亲水性 ${ }^{[12]}$. 树脂的类型对砷的去除效果有很大影响, $201 \times 7$ 强碱性苯乙烯系阴离子交换树脂以季胺基为主 要活性基团,季铵基连接有 3 个甲基, 而 D301 大孔弱碱阴离子交换树脂以叔胺基为活性基团,叔胺基连接 2 个甲基, 因此对 $\mathrm{H}_{2} \mathrm{AsO}_{4}^{-}$以及 $\mathrm{HAsO}_{4}^{2-}$ 的亲和能力较弱. 并且大孔结构树脂的选择性小于凝胶型树脂, 这种选 择性在稀溶液中较大, 在浓溶液中较小 ${ }^{[13]}$, 因此树脂 I 较树脂 II 对泉涌水中的砷具有较大的交换吸附容量.

$\mathrm{pH}$ 值对 $\mathrm{As}(\mathrm{V})$ 的去除效果同样有着较大的影响. 有研究发现, 在 $\mathrm{pH}$ 值较低时, 砷酸根离子主要以 $\mathrm{H}_{3} \mathrm{AsO}_{4}$ 中性分子存在, 很难发生离子交换反应; 随着 $\mathrm{pH}$ 值的升高, 溶液中的 $\mathrm{H}_{2} \mathrm{AsO}_{4}^{-} 、 \mathrm{HAsO}_{4}^{2-} 、 \mathrm{AsO}_{4}^{3-}$ 形态占 优势, 可使交换吸附容量增大; 当 $\mathrm{pH}>7$ 时, $\mathrm{OH}^{-}$可能与砷酸根离子竞争表面交换位使得吸附量下降. 所以, 去除砷酸根离子的适宜 $\mathrm{pH}$ 值为 $3.5 \sim 7.0^{[14]}$.

此外有研究表明, 进水中高浓度的 $\mathrm{SO}_{4}^{2-}(>120 \mathrm{mg} / \mathrm{L}) 、 \mathrm{NO}_{3}^{-} 、 \mathrm{Cl}^{-} 、 \mathrm{TDS}(>1000 \mathrm{mg} / \mathrm{L})$ 也会与 $\mathrm{As}(\mathrm{V})$ 形成 竞争效应而导致离子交换失效 ${ }^{[12]}$. 因此, 离子交换除砷技术适合于较为洁净、背景离子强度较小的水体. 阳 宗海泉涌水含硫浓度低于 $50 \mathrm{mg} / \mathrm{L}$, 故对离子交换干扰较小.

\section{3 结论}

1) 小试试验表明, 树脂 I 、II 均可用于处理阳宗海泉涌水, 在较小流量/树脂床层比条件下, 处理出水总 砷浓度 $<0.10 \mathrm{mg} / \mathrm{L}$, 可达《农田灌溉水水质标准》( GB 5084-1992), 树脂 I 较树脂 II 对泉涌水中的砷具有较 大的交换吸附容量, 使用周期更长.

2) 在出水总砷浓度 $<0.10 \mathrm{mg} / \mathrm{L}$ 的时段内, 小试试验和中试实验中平均每克树脂 I 去除砷分别为 2.101 和 $2.020 \mathrm{mg}$, 两者结果基本吻合,故可利用离子交换法进行扩大规模处理含砷废水.

3) 按中试规模估算运行费用: 每吨水电耗约为 $75 \mathrm{~W} \cdot \mathrm{h}$, 按普通工业电度电价标准 0.898 元 $/(\mathrm{kW} \cdot \mathrm{h})$, 折合电费约为每吨水 0.06 元; 每吨水再生液耗费约为 0.65 元; 反洗、配液用清水直接来自处理尾水, 不产生 直接费用; 选择性复合树脂可以经过再生继续利用, 且制作成本低廉, 处理运行成本较低.

4) 实际应用中可以根据出水浓度要求控制吸附反应时间来保证出水水质达标.

\section{4 参考文献}

[ 1 ] Liu Wenju, Zhu Yongguan, Hu Ying et al. Effects of arsenic from soil and irrigation-water on as accumulation on the root 
surfaces and in mature rice plants. Environmental Science, 2008, 29(4): 862-868 (in Chinese with English abstract). DOI : 10.13227/j.hjkx.2008.04.043. [刘文菊, 朱永官, 胡荣等. 来源于土壤和灌溉水的砷在水稻根表及其体内的富 集特性. 环境科学, 2008, 29(4):862-868.]

[ 2 ] Bo Ying, Luo Liqiang. Geochemical characteristics and research direction of arsenic. Rock and Mineral Analysis, 2009,28 (6) : 569-575 (in Chinese with English abstract). [伯英, 罗立强. 砷的地球化学特征与研究方向. 岩矿测试, 2009, $28(6)$ : 569-575.]

[ 3 ] Huang Xin, Gao Naiyun, Liu Cheng et al. Review on arsenic removal technologies from drinking water. Water Purification Technology, 2007, 26(5): 37-41 (in Chinese with English abstract). [黄金鍂, 高乃云, 刘成等. 饮用水除砷工艺研究 进展. 净水技术, 2007, 26(5): 37-41.]

[ 4 ] Liao Min, Xie Zhengmiao, Wang Rui. A primary study on removal of arsenic in wastewater by arsenic-resistant helotism. Environment Pollution \& Control, 1997, 19(2): 11-12, 38(in Chinese with English abstract). [廖敏, 谢正苗, 王锐. 菌藻共生体去除废水中砷初探. 环境污染与防治, 1997, 19(2) : 11-12, 38.]

[ 5 ] Xiong Ruyi, Song Weifeng. Arsenic pollution in environment and its trend of treated technology. Guangdong Chemical Industry, 2007, 34(11) : 92-94(in Chinese with English abstract). [ 熊如意, 宋卫锋. 环境砷污染及其治理技术发展趋 势. 广东化工, 2007, 34(11): 92-94.]

[ 6 ] Hu Tianjue, Zeng Guangming, Chen Weiping et al. Study on selective adsorption removal and recovery of As( III ) in waste solution containing As (III) with chelating resin containing mercapto groups. Journal of Hunan University, 1998, 25(6) : 75-80 (in Chinese with English abstract). [胡天觉, 曾光明, 陈维平等. 选择性高分子离子交换树脂处理含砷废水. 湖南大学学报, 1998, 25(6): 75-80.]

[ 7 ] Wang Zhenyu, Liu Jiadi, Li Zongzhan et al. Research on the removal of metal impurity ions from the recycling water of gold concentrator with ion-exchange resins. Gold, 2010, 31(2): 48-51 (in Chinese with English abstract). [王振玉, 刘 家弟，李宗站等. 离子交换树脂去除金矿选矿循环用水中金属杂质离子的研究. 黄金, 2010, 31(2): 48-51.]

[ 8 ] Xiao Yabing, Qian Shahua, Huang Ganquan et al. Adsorption properties of nanometer-size $\mathrm{TiO}_{2}$ for $\mathrm{As}(\mathrm{III})$ and $\mathrm{As}(\mathrm{V})$. Journal of Analytical Science, 2003, 19(2) : 172-174 (in Chinese with English abstract). [肖亚兵, 钱沙华, 黄洤泉等. 纳米二氧化钛对砷 (III) 和砷 (V) 吸附性能的研究. 分析科学学报, 2003, 19(2): 172-174.]

[ 9 ] Ti Yun, Zhang Xu, Tong Di et al. Harmfulness of arsenic pollution and techniques of arsenic removal. Liaoning Chemical Industry, 2008, 37(9): 629-631 (in Chinese with English abstract). [提芸, 张旭, 佟迪等. 砷污染的危害及除砷方 法探讨. 辽宁化工, 2008, 37(9): 629-631.]

[10] Chen Jingjun, Jiang Boquan, Wang Wei. Status and progress of arsenic removal. Jiangxi Chemical Industry, 2004, (2) : 1-4 (in Chinese with English abstract). [陈敬军, 蒋柏泉, 王伟. 除砷技术现状与进展. 江西化工, 2004, (2) : 1-4. ]

[11] Ding Liang, Chen Huanxin, Huang Chengwu. Introduction about the removal of arsenic from groundwater in Albuquerque, America. Endemic Diseases Bulletin, 2001, 16(2) : 108-110(in Chinese with English abstract). [丁亮, 陈焕新, 黄承 武. 美国 Albuquerque 市的地下水除砷方法介绍. 地方病通报, 2001，16(2)：108-110.]

[12] Yao Juanjuan, Gao Naiyun, Xia Shengji et al. Research progress on arsenic removal from drinking water. Industrial Water \& Wastewater, 2007, 38(4) : 1-5(in Chinese with English abstract). [姚娟娟, 高乃云, 夏圣骥等. 饮用水除砷技术 研究新进展. 工业用水与废水, 2007, 38(4) : 1-5.]

[13] Qu Liu. Reaserch of ion exchange polymer materials. China Science and Technology Information, 2010, (1): 128-129(in Chinese). [曲柳. 离子交换高分子材料研究. 中国科技信息, 2010，(1)：128-129.]

[14] Liu Ruixia, Wan Yaxiong, Tang Hongxiao. Removal of arsenate by a new type of ion exchang fiber. Environmental Science, 2002, 23(5) : 88-91 (in Chinese with English abstract). [刘瑞霞, 王亚雄, 汤鸿霄. 新型离子交换纤维去除水中砷 酸根离子的研究. 环境科学, 2002, 23(5): 88-91.] 